regions, especially in the North (91.2\%) and Northeast (87.1\%). Of the 1066842 deaths registered in 2008, almost 60\% had causes related to the circulatory system, cancer and respiratory tract. The SMR for diseases of the circulatory system was 139.5 deaths per 100000 inhabitants, highest in the Southeast (143.2) and lowest in the North (113.8). For neoplasms, the SMR was 75.5 deaths per 100000 inhabitants, higher in the South (92.6) and lowest in the North (57.7).

Conclusion From 1980 to 2008, there was a change in the mortality profile, with increased proportion of deaths due to causes related to chronic diseases, to the detriment of infectious and parasitic diseases. The social and economic differences between Brazilian regions are reflected in differences in mortality rates.

\section{P2-94 TYPES OF SMOKERS, DEPRESSION AND DISABILITY IN TYPE 2 DIABETES: A LATENT CLASS ANALYSIS}

doi:10.1136/jech.2011.142976i.29

${ }^{1} \mathrm{G}$ Gariepy, ${ }^{*} \mathrm{~A}$ Malla, ${ }^{2} \mathrm{~J}$ Wang, ${ }^{3} \mathrm{~L}$ Messier, ${ }^{3} \mathrm{I}$ Strychar, ${ }^{3} \mathrm{~A}$ Lesage, ${ }^{1} \mathrm{~N}$ Schmitz. ${ }^{1}$ Douglas Mental Health University Institute, Montreal, Quebec, Canada; ${ }^{2}$ University of Calgary, Calgary, Alberta, Canada; ${ }^{3}$ University of Montreal, Montreal, Quebec, Canada

Despite the detrimental effects of smoking on health, a high number of adults with type 2 diabetes continue to smoke. Identifying distinct profiles of smokers could help tailor smoking intervention programs in this population and may help uncover high risk subgroups with unfavourable health outcomes. This study examined whether smokers with type 2 diabetes could be classified into different profiles based on socioeconomic characteristics, smoking habits and lifestyle factors. Depression and disability outcomes were compared across smoking profiles. A community sample of adults with self-reported diabetes was selected from random digit dialing. Analyses included 383 participants with type 2 diabetes who were current smokers. Participants were interviewed at baseline (2008) and re-interviewed 1 year later (2009). Latent class analysis was used to identify types of smokers. We uncovered three meaningful classes of smokers: (1) long-time smokers with long-standing diabetes $(n=105)$, (2) heavy smokers with deprived socioeconomic status, poor health and unhealthy lifestyle characteristics $(n=105)$, (3) working and active smokers, recently diagnosed with diabetes $(n=173)$. Members of class 2 were significantly more likely to be disabled and depressed at baseline and follow-up compared with others. They were also less likely to have quit smoking at follow-up, despite attempting to quit as often as others. Different profiles of smokers exist among adults with type 2 diabetes. One class of smokers is particularly linked with depression, disability and a deprived socioeconomic situation. Distinguishing between types of smokers may enable clinicians to tailor their approach to smoking cessation.

\section{P2-95 OBESITY INCREASES 28\% IN 3 YEARS IN PREMENOPAUSAL LOW-INCOME CHILEAN WOMEN INDEPENDENTLY OF BODY SIZE MISPERCEPTION}

doi:10.1136/jech.2011.142976i.30

${ }^{1} \mathrm{M}$ L Garmendia, ${ }^{*} \mathrm{~F}$ Alonso, ${ }^{2} \mathrm{~J}$ Kain, ${ }^{1} \mathrm{C}$ Corvalan, ${ }^{1} \mathrm{M}$ de Aguirre, ${ }^{1} \mathrm{~J}$ Searle. ${ }^{1}$ School of Public Health, Faculty of Medicine, Universidad de Chile, Santiago, Metropolitan Region, Chile; ${ }^{2}$ Institute of Nutrition and Food Technology, Universidad de Chile, Santiago, Metropolitan Region, Chile; ${ }^{3}$ Faculty of Medicine, Universidad de Chile, Santiago, Metropolitan Region, Chile

Introduction How body image perception (BIP) influences changes in Body Mass Index (BMI) in adult women has not been evaluated.

Objective To assess BMI-changes over a 3-year period and their relationship with BIP in a Chilean women population-based cohort.
Methods Weight and height were measured at baseline (32.0 $\pm 7.0 \mathrm{y})$ and at follow-up in 510 women participants in the GOCS study. BMI was used to classify women in normal $\left(\leq 25 \mathrm{~kg} / \mathrm{m}^{2}\right)$, overweight $\left(>25 \mathrm{~kg} / \mathrm{m}^{2}\right)$, or obese $\left(>30 \mathrm{~kg} / \mathrm{m}^{2}\right)$. BIP was self-assessed at baseline using the Stunkard Figure Rating Scale (nine figures from very thin to very obese). BIP-discrepancy was defined as the disagreement between measured-BMI and assigned-BMI of the selected figure (defined on a previous validation study). Multivariate linear regression was used to assess the influence of BIP on BMI-changes stratifying by nutritional status and controlling for potential confounders (ie, parity, schooling, age).

Results At baseline, 61\% had excess weight (37\% overweight and $24 \%$ obese). In 3 years, this number increased to $70 \%$ (38\% overweight and $31 \%$ obese). One out of 4 increased their BMI category, particularly normal women $(28.9 \%$ from normal to overweight and $22.5 \%$ from overweight to obesity). At baseline, BIP-discrepancy was $66 \%$ and was associated to concurrent BMI only in obese (p-forinteraction $<0.05$; coefficient all sample $=0.48 ; 95 \% \mathrm{CI}-0.49$ to 1.45 ; coefficient $_{\text {obese }}=1.65,95 \%$ CI 0.03 to 3.28). BIP-discrepancy was unrelated to the 3 -year changes in BMI ( $\mathrm{p}$-for-interaction $>0.05$, coefficient $=-0.45,95 \% \mathrm{CI}-0.95$ to 0.04 ).

Conclusion In 3 years we observe a large BMI increase among young women of a post-transitional country. Body size misperception does not explain this large increase. Population strategies are needed to stop this detrimental trend.

Funding Fondecyt1090252.

\section{P2-96 CAN INCREASING PHYSICIAN FEE-FOR-SERVICE PAYMENTS IMPROVE SERVICE REGULARITY IN ELDERLY PATIENTS WITH CHRONIC DISEASE?}

doi:10.1136/jech.2011.142976i.31

${ }^{1} \mathrm{D}$ Gibson, ${ }^{1,2} \mathrm{R}$ Moorin, 'D Preen, ${ }^{1} \mathrm{~J}$ Emery, ${ }^{1} \mathrm{D}$ Holman. ${ }^{1}$ University of Western Australia, Perth, Western Australia, Australia; ${ }^{2}$ Curtin University, Perth, Western Australia, Australia

Introduction Developed nations are experiencing increased burden on their health systems from chronic diseases. One avenue Australia is seeking to reduce this burden is via primary care services with increased financial incentives for physicians to provide more continuous care to elderly patients, especially those suffering from chronic diseases. This study assessed the influence of increased feefor-service values on regularity and frequency of primary care visits, and examined the independent effects of demographic factors (age and gender) and chronic disease history.

Methods We performed a retrospective, population-based, longitudinal cohort study, linking routinely collected primary care service claims and hospital separation data in people aged 65 or more years in Western Australia from 2001 to 2006. Polytomous logistic regressions evaluated changes in the likelihood of increased primary care service regularity and frequency in exposed and unexposed individuals, adjusting for age, gender and recent chronic disease history. Results The higher value services significantly and substantially increased relative likelihood (up to 14 times) of higher regularity with no corresponding higher frequency of primary care services. Increased regularity was more likely to occur with increasing age, except in the oldest age group $(90+$ years). Some chronic disease histories (eg, diabetes) conferred a higher likelihood of improved regularity than others (eg, hypertension).

Conclusion The study suggests a potential for modification of physician and patient behaviour using incentivises within the current fee-for-service system in Australia. This type of incentive could be adopted in systems, which are not based on fee-for-service, as an additional incentive or bonus payment. 